\title{
Manejo Nutricional en Niños y Adolescentes con Fibrosis Quística
}

\author{
SALESA BARJA Y. ${ }^{1}$, MARÍA JESÚS REBOLLO G. ${ }^{2}$ \\ 1. Médico Nutriólogo. Departamento de Pediatría. Pontificia Universidad Católica de Chile. Hospital Josefina Martínez \\ (ex CEDERI). \\ 2. Médico Nutriólogo. Servicio de Pediatría. Complejo Hospitalario San Borja Arriarán.
}

\begin{abstract}
Nutritional Management of Children with Cystic Fibrosis: Review and Practical Applications
\end{abstract}

The importance of optimal nutrition has been well demonstrated in Cystic Fibrosis patients; it affects their mortality, diminishes morbidity and could decrease the deterioration of pulmonary function. However, success of nutritional treatment depends mostly of its early onset. Assistance by enteral route as well as gastrostomy in early stages becomes important since early detection of nutritional deficits and active support result in better response to treatment and slower deterioration of pulmonary function. With an increased survival, new nutritional challenges appear, like deficits of specific EFA, micronutrients, minerals, and possible antioxidant and anti-inflammatory effects of various nutrients. The object of this article is to update nutritional treatment of children and adolescent CF patients.

(Key words: Cystic fibrosis, under nutrition, nutrition support).

Rev Chil Pediatr 2009; 80 (3): 274-284

\section{RESUMEN}

La importancia de un óptimo estado nutricional ha sido bien demostrada en los pacientes con Fibrosis Quística; incide en la mortalidad, disminuye la morbilidad y podría favorecer un menor deterioro de la función pulmonar. Sin embargo, el éxito del tratamiento nutricional depende en gran medida de la prontitud con que se realice, ya que la detección precoz del déficit nutricional favorece una mejor respuesta al tratamiento, a la vez que una actitud más activa favorece un menor deterioro, de manera que tanto el apoyo por vía enteral como la indicación de gastrostomía en etapas menos tardías cobran mayor relevancia. Con el aumento de la sobrevida se presentan además nuevos desafíos nutricionales, como son déficits específicos de AGE, micronutrientes y minerales, al igual que efectos antioxidantes y antiinflamatorios de diversos nutrientes. En esta revisión se espera haber entregado un conocimiento actualizado y aportado algunas herramientas para el manejo nutricional de los niños con FQ.

(Palabras clave: Fibrosis quística, apoyo nutricional, desnutrición, déficit de macro nutrientes y micro nutrientes).

Rev Chil Pediatr 2009; 80 (3): 274-284

Trabajo recibido el 19 de agosto de 2008, devuelto para corregir el 21 de enero de 2009, segunda versión el 30 de marzo de 2009, aceptado para publicación el 24 de abril de 2009.

Correspondencia a:

Dra. Salesa Barja Y.

E-mail: sbarja@puc.cl 


\section{Introducción}

La mejoría en la sobrevida de los pacientes con Fibrosis Quística (FQ) ha sido consecuencia de la interacción de diferentes factores, como son el manejo del paciente en grupos interdisciplinarios de salud o "clínicas de FQ", la mejoría en las técnicas de drenaje de vía aérea, el uso de antibióticos de amplio espectro, el diagnóstico precoz mediante tamizaje neonatal y particularmente, la mejoría del tratamiento nutricional ${ }^{1}$. En el año 2006 la edad media de sobrevida reportada en 24000 pacientes por la Fundación de Fibrosis Quística (CFF) en EEUU, fue de 37 años $^{2}$, mientras que en Chile se estima que está entre los 12 y 18 años de edad ${ }^{3}$.

El estado nutricional normal se asocia a mayor sobrevida y a mejor función pulmonar en los pacientes con FQ, a la vez que la desnutrición se asocia a mayor mortalidad ${ }^{4,5}$, incluso en forma independiente del deterioro de la función pulmonar, propia de la evolución natural de la enfermedad ${ }^{6}$. Por ello, se han desarrollado consensos para optimizar y homogeneizar su tratamiento nutricional ${ }^{7-9}$, los cuales coinciden en que el enfoque debiera ser anticipatorio, oportuno y eficaz ${ }^{10}$. En forma complementaria, el equipo de salud debe considerar la individualidad de cada niño y de su entorno familiar, a la vez que educar y estimular la autonomía y participación de ambos ${ }^{11}$ para favorecer una mejor evolución de la enfermedad y mejorar su calidad de vida.

En Chile, el año 2001 se realizó un Consenso Nacional de $\mathrm{FQ}^{3}$ que ha servido de guía para el manejo integral de estos pacientes, a partir de 2008 está también disponible la Norma Técnica para el manejo nutricional y gastroenterológico de la Fibrosis Quística en Chile, fruto del trabajo colaborativo de un grupo de nutriólogos y gastroenterólogos de diferentes centros hospitalarios y universitarios de nuestro país ${ }^{12}$.

El objetivo del tratamiento nutricional es lograr un crecimiento y desarrollo comparables al de los niños sanos; es notable que aunque en los últimos 15 años los pacientes registrados en la CFF hayan mejorado el promedio de IMC en 5 a 20 puntos percentilares en diferentes edades, mantienen una declinación después de los 9 años (figura 1). Así, pese a la mejoría lograda, la prevalencia de desnutrición es aún significativa: en 2005 el $23 \%$ de los pacientes tenía un peso menor al percentil $10^{2,4}$. Dado el impacto del IMC sobre la función pulmonar (figura 2), el objetivo actual de la CFF es lograr mantener a estos pacientes sobre el percentil 50 de $\mathrm{IMC}^{4}$. En Chile, el 25\% de los pacientes registrados en el Programa Nacional de FQ presenta desnutrición en cuanto al peso para la talla o la edad. En una muestra de 33 pacientes de un centro universitario de Santiago, $18 \%$ presentaba IMC menor a Pc10 (Observaciones no publicadas), menor a la reportada en una casuística de Brasil en 127 pacientes, de $41 \%{ }^{13}$.

Diferentes factores favorecen la desnutrición de estos niños (tabla 1), pero en presencia de una adecuada suplementación de enzimas pancreáticas, tanto en dosis como en implementación práctica y adherencia, los mecanismos más frecuentes son la ingesta insuficiente de

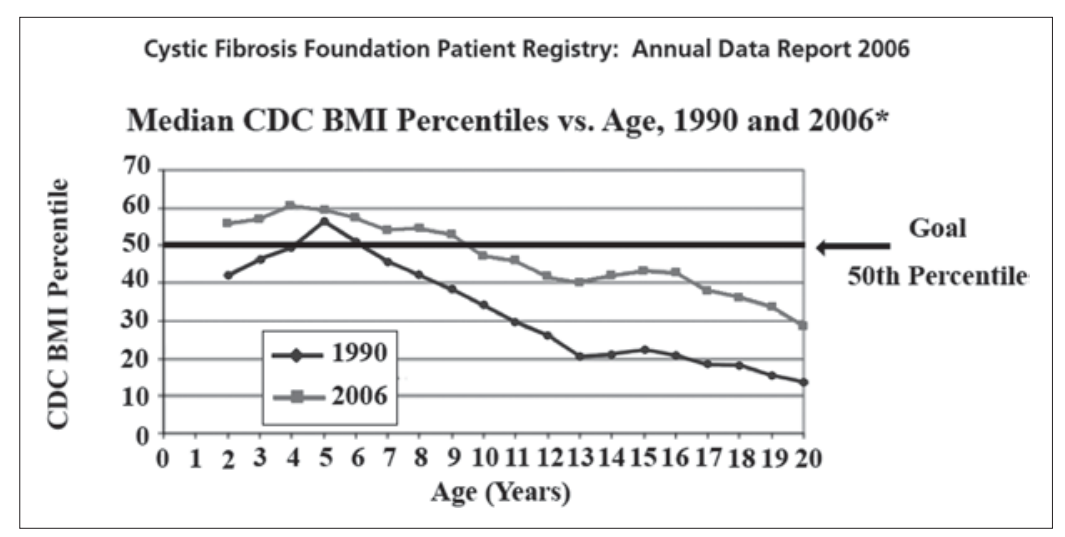

Figura 1. Cambios en la mediana del IMC de acuerdo a la edad, en niños con FQ, entre los años 1990-2006 (EE.UU., CFF). 


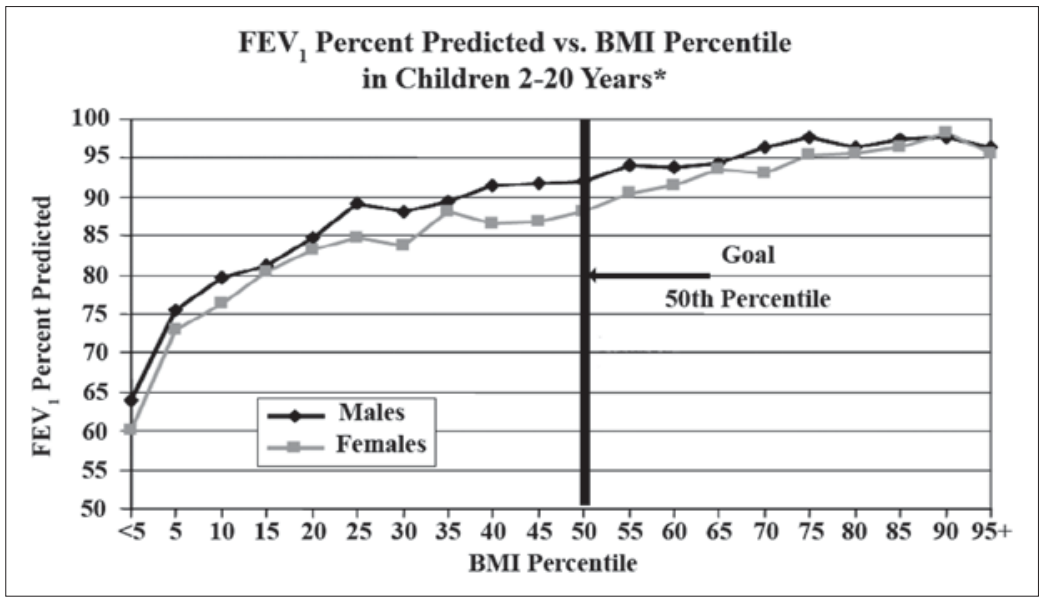

Figura 2. Relación entre estado nutricional y función pulmonar en niños con Fibrosis Quística (Cystic Fibrosis Foundation Patient Registry: Annual Data Report 2006).
Tabla 1. Mecanismos de desnutrición en pacientes portadores de Fibrosis Quística

\begin{tabular}{|c|c|}
\hline Mecanismos & Causas \\
\hline \multirow[t]{2}{*}{$\downarrow$ Ingesta } & $\begin{array}{l}\text { Anorexia (Enfermedad crónica, reagudi- } \\
\text { zaciones, medicamentos) }\end{array}$ \\
\hline & $\begin{array}{l}\text { Disfagia (Reflujo gastroesofágico, eso- } \\
\text { fagitis, sonda naso-gástrica) } \\
\text { Dificultad respiratoria } \\
\text { Rechazo, aversión, trastorno de la con- } \\
\text { ducta alimentaria } \\
\text { Aporte insuficiente }\end{array}$ \\
\hline$\uparrow$ Requerimientos & $\begin{array}{l}\text { Infección, inflamación, stress } \\
\text { Mayor trabajo respiratorio } \\
\text { Aumento del gasto energético en reposo }\end{array}$ \\
\hline$\uparrow$ Pérdidas & $\begin{array}{l}\text { Pérdidas fecales, esteatorrea } \\
\text { Vómitos (Reflujo gastroesofágico, tos) } \\
\text { Expectoración (proteínas) } \\
\text { Renales (glucosuria, proteinuria) }\end{array}$ \\
\hline$\downarrow$ Utilización & $\begin{array}{l}\text { Hipoxemia crónica, } \uparrow \text { metabolismo anae- } \\
\text { róbico, acidosis. }\end{array}$ \\
\hline
\end{tabular}

alimentos y el aumento del gasto energético ${ }^{8}$. La anamnesis nutricional detallada permite identificar precozmente estos aspectos, sin embargo, el trabajo colaborativo de los miembros del equipo de salud ayuda a valorar la importancia relativa que distintos factores pueden tener en un mismo paciente y en diferentes etapas de la evolución de su enfermedad.

Este artículo se focaliza en la oportunidad y aplicación del tratamiento nutricional, utilizándose como referencia principal la Norma Técnica antes referida ${ }^{12}$.
Tabla 2. Frecuencia de evaluaciones nutricionales* (Norma Técnica FQ, MINSAL 2008)

\begin{tabular}{lccc}
\hline Medición & $\begin{array}{c}\text { Al diag- } \\
\text { nóstico }\end{array}$ & $\begin{array}{c}\text { Cada 3 } \\
\text { meses }\end{array}$ & $\begin{array}{c}\text { Semes- } \\
\text { tral }\end{array}$ \\
\hline Peso & $X$ & $X$ & \\
Longitud/Estatura & $X$ & $X$ & \\
$\begin{array}{l}\text { P. Craneano (hasta } \\
\text { los } 2 \text { años) }\end{array}$ & $X$ & $X$ & \\
$\begin{array}{l}\text { P. Braquial } \\
\text { Pliegues cutáneos }\end{array}$ & $X$ & $X$ & \\
Maduración puberal & & $X$ & \\
Evaluación de ingesta (24 h) & $X$ & & $X$ \\
Guía anticipatoria dietaria & & & $X$ \\
y de hábitos alimentarios & $X$ & $X$ & \\
\hline
\end{tabular}

* La consignada es la frecuencia mínima. En períodos críticos, de mayor riesgo o de crecimiento insatisfactorio, los controles deben ser mensuales. La evaluación será realizada por médico especialista en nutrición o pediatra general al diagnóstico y después, cada 3 meses, puede ser alternada con nutricionista.

\section{Detección precoz del deterioro nutricional}

La intervención nutricional debe ser precoz, para lo cual es necesario realizar controles clínicos regulares y adelantarse al deterioro del crecimiento, aplicando medidas proporcionales y progresivas. La detección del déficit nutricional es clínica, ya que los exámenes de laboratorio suelen afectarse tardíamente, habiéndose acordado una frecuencia de controles que puede aumentar si el paciente lo requiere (tabla 2). 
Es necesario considerar que existen períodos críticos de mayor riesgo nutricional: En primer lugar, los primeros 12 meses después de hecho el diagnóstico de FQ o el primer año de vida en lactantes diagnosticados precozmente. En segundo lugar, el período peri-pubertad ( 9 a 16 años en mujeres y 12 a 18 años en hombres) caracterizado por un rápido crecimiento, alta demanda energética y menor adherencia al tratamiento ${ }^{8}$. Por último, durante las exacerbaciones y/o sobreinfecciones, en que aumentan los requerimientos y las pérdidas, a la vez que disminuye la ingesta. A la inversa, en los períodos de tratamiento efectivo y buen control broncopulmonar, se puede lograr un mejor rendimiento de la intervención nutricional.

En la evaluación clínica se calculan los índices antropométricos y se analizan en forma integrada: En los menores de 1 año de edad se utiliza el índice Peso/Edad (P/E) por su mayor sensibilidad para detectar desnutrición. El índice Talla/Edad (T/E) se afecta si ésta tiene mayor tiempo de evolución y debe valorarse en relación a la carga genética de estatura parental. Los índices de proporcionalidad del peso para la talla son el índice Peso/Talla $(\mathrm{P} / \mathrm{T}=$ [peso actual/peso ideal] x 100) para niños de hasta 6 años y el índice de masa corporal (IMC $=[$ peso/ talla $\left.{ }^{2}\right]$ ) en los mayores. La evidencia indica que el IMC es un marcador sensible y de mayor correlación con la evolución de la función pulmonar en los niños con FQ mayores de 2 años ${ }^{4,14}$.

Es recomendable expresar los mencionados índices como porcentaje de la mediana o como score-z, y no como percentiles o rangos de desviación estándar, ya que éstos no permiten visualizar variaciones, en especial en rangos extremos. También considerar que el graficar las mediciones secuencialmente permite observar cambios con mayor facilidad. Si es posible, complementar los índices con estimadores clínicos de masa corporal magra y grasa, los cuales pueden aumentar su sensibilidad para detectar desnutrición.

\section{Déficits nutricionales}

El crecimiento deficiente es con frecuencia fruto de una baja ingesta energética, a la vez que se ha demostrado que un aporte adecuado favorece una mejor evolución ponderal ${ }^{4}$. Sin embargo, se deben considerar tanto los déficits de macronutrientes como de micronutrientes, ya que ambos influyen directa o indirectamente el progreso pondo-estatural ${ }^{10}$.

Los requerimientos energéticos son mayores en una alta proporción de los pacientes ${ }^{4}$, de manera que las recomendaciones habituales están sobre $120 \%$ de aquellas para niños sanos (FAO-OMS: RDI 2002). Los requerimientos tienen estrecha relación con la gravedad de la enfermedad $^{15}$, siendo comparables a los de niños sanos en pacientes eutróficos, con compromiso pulmonar leve y en períodos estables de la enfermedad ${ }^{8,16}$. Por otra parte, existe un equilibrio entre el aumento del gasto, ingesta necesaria, control de pérdidas, utilización y adaptación metabólica del paciente ${ }^{10,17}$. La estimación de las necesidades de energía mediante fórmulas matemáticas específicas no ha sido suficientemente evaluada en la clínica ${ }^{18}$ aunque la fórmula propuesta en el consenso de $1992^{7}$ se utiliza con frecuencia en el ámbito clínico (figura 3). Siempre debe considerarse cada paciente individualmente, teniendo presente que aunque los niños con FQ alcancen ingestas elevadas de alimentos, muchas veces no logran las metas propuestas ${ }^{19}$. Por ello, es conveniente asegurar una ingesta suficiente, a la vez que evitar también el aporte excesivo, con el consiguiente sobrepeso y siempre considerar el grado de actividad física que el paciente realiza.

El aporte proteico debe garantizar las recomendaciones diarias de ingesta (RDI 2002), requiriéndose aportes mayores en niños pequeños $y / o$ con déficit nutricional ${ }^{9}$, considerándose tanto la edad como el estado catabólico del paciente. En relación a grasas, se recomienda que sea el $35-45 \%$ del aporte calórico total ${ }^{5,7-9}$, en especial el 3-5\% de éste como ácidos grasos esenciales (AGE). Se ha reportado alteración en el perfil de ácidos grasos en los pacientes con FQ, especialmente una disminución del ácido Linoleico (AL) y del ácido Docosahexanoico $(\mathrm{ADH})$, a la vez que un aumento del ácido Araquidónico (AA). Esta situación se debería no sólo a ingesta insuficiente o malabsorción, ya que se ha documentado déficit en pacientes sin insuficiencia pancreática. Estudios en cultivos celulares han mostrado una disfunción del 


\begin{tabular}{|c|c|c|c|}
\hline \multicolumn{4}{|c|}{$\begin{array}{l}\text { GET = GER (fórmula OMS) x } \\
\text { (Coef. actividad física + Coef. enfermedad) x Función pancreática } \\
\text { Fórmulas para el cálculo de Gasto energético en reposo (GER), OMS } 1985 .\end{array}$} \\
\hline \multirow{2}{*}{$\begin{array}{l}\text { Edad } \\
0-3 \text { años }\end{array}$} & Hombre & \multicolumn{2}{|l|}{ Mujer } \\
\hline & $(60,9 \times$ Peso $)-54$ & $(61 \times$ Peso $)$ & -51 \\
\hline $3-10$ años & $(22,7 \times$ Peso $)+495$ & $(22,5 \times$ Peso $)$ & +499 \\
\hline 10 a 18 años & $(17,5 \times$ Peso $)+651$ & $(12,2 \times$ Peso $)$ & +746 \\
\hline \multicolumn{4}{|c|}{$\begin{array}{l}\text { Coeficiente de actividad: } \\
\text { En cama }(1,3) \text {, sedentario }(1,5) \text { y activo }(1,7)\end{array}$} \\
\hline \multicolumn{4}{|c|}{$\begin{array}{l}\text { Coeficiente de enfermedad: De acuerdo al } \mathrm{VEF}_{1} \\
\mathrm{VEF}_{1}>80 \%=0, \mathrm{VEF}_{1} 40-79 \%=0,2, \mathrm{VEF}_{1}<40 \%=0,3 \text {, muy severo }=0,5\end{array}$} \\
\hline \multicolumn{2}{|c|}{ Ajuste por función pancreática: } & \multicolumn{2}{|c|}{ Sin insuficiencia (x 1), Con insuficiencia (x 0.93/tasa grasa fecal) } \\
\hline & Aproximación $=0$ & & \\
\hline
\end{tabular}

Figura 3. Cálculo de requerimientos energéticos en pacientes con Fibrosis Quística. Adaptado de: Ramsey BW y cols. $1992^{7}$. gen CFTR asociada con alteración en el metabolismo de los ácidos grasos ${ }^{20}$. Existe evidencia que este déficit tendría asociación con el curso clínico de la enfermedad; estudios muestran que el score de Schawman presenta correlación positiva con niveles plasmáticos de $\mathrm{AA} y$ $\mathrm{AL}$, estos últimos serían un buen marcador del estatus de AGE y se asocian en forma directa al crecimiento, composición corporal y función pulmonar $^{21,22}$.

Por otro lado, la ingesta de ácidos grasos omega 3: DHA y Eicosapentanoico (EPA) puede tener efectos en disminuir el estado proinflamatorio de estos pacientes, si bien la mayoría de los estudios muestran un aumento de los niveles de AGE suplementados, ello no tiene una repercusión clínica significativa, por lo que se requieren estudios multicéntricos y a largo plazo para recomendar una suplementación sistemática ${ }^{23-25}$.

Pueden existir deficiencias de vitaminas, las cuales se han evidenciado con la mayor sobrevida y mejoría global de los pacientes. Aún con el aporte adecuado puede existir déficit subclínico, de modo que se recomienda monitorizar anualmente niveles plasmáticos, ya que la expresión clínica de los déficits de micronutrientes es habitualmente tardía. Dentro de las vitaminas liposolubles, considerar déficit de vitamina $\mathrm{D}$ si existe poca exposición solar y en especial en presencia de daño hepatocelular. Se han reportado bajas concentraciones plasmáticas de $25(\mathrm{OH}) \mathrm{D}$ a pesar de recibir adecuadas dosis en un alto porcentaje de niños y adolescentes con $\mathrm{FQ}^{26}$. Se ha demostrado además una prevalencia creciente de enfermedad ósea en los adultos con FQ, caracterizada $\mathrm{x}$ osteopenia en el 50-75\% de ellos y fracturas costales o de aplastamiento vertebral ${ }^{27}$, de manera que actualmente se recomienda control anual con densitometría ósea a partir de los 10 años de edad ${ }^{12,28}$. Otros factores no nutricionales como la susceptibilidad genética, retraso puberal o estado inflamatorio participan en su génesis multifactorial ${ }^{29}$. La vitamina $\mathrm{K}$ es cofactor de la osificación y junto al déficit de vitamina $\mathrm{D}$ puede tener un rol importante en la osteopenia asociada a $\mathrm{FQ}^{30}$. Por el stress oxidativo aumentado propio de las enfermedades crónicas, de las infecciones recurrentes y colonización bacteriana, puede existir mayor consumo de vitaminas antioxidantes A y E, cuyos niveles disminuidos, aún dentro de rangos normales, se asocian a mayor grado de exacerbaciones pulmonares ${ }^{31}$.

La expresión clínica del déficit de minerales 
es tardía, en cuanto a osteopenia, se ha planteado que también puede existir baja ingesta o absorción deficiente de Calcio, secundaria a déficit de vitamina $\mathrm{D}$, a mayor acidez intestinal o a pérdida fecal con sales biliares cuando existe esteatorrea ${ }^{32}$. La anemia es de prevalencia variable: $30 \%$ en niños y $50 \%$ en adultos con FQ, su etiología principal es el déficit de Hierro, ya que hay menor ingesta y utilización, al igual que mayor consumo y pérdidas por tracto digestivo y secreciones bronquiales, lo cual podría favorecer el desarrollo de Pseudomona Aeruginosa ${ }^{33}$. Se ha reportado déficit subclínico de Zinc en 30\% de los pacientes, producto de una mayor utilización, menor absorción intestinal o de pérdidas fecales, sin embargo, aunque no está clara su repercusión clínica $^{34}$, se ha postulado que la suplementación con Zinc podría tener efecto antiinflamatorio ${ }^{35}$. Puede existir menor absorción de Magnesio en tratamientos prolongados con aminoglicósidos ${ }^{36}$, habiéndose postulado que su suplementación podría mejorar la respuesta a DNAsa inha$\operatorname{lada}^{37}$.

\section{Oportunidad de la intervención nutricional}

Si bien cada paciente es evaluado en forma particular y la conducta debe ser tomada considerando los factores que favorecen la desnutrición, se ha planteado una actitud más activa en el apoyo nutricional, con el fin de mejorar la evolución de la enfermedad ${ }^{4}$ y la calidad de vida del paciente. Al adaptar de manera más exigente el consenso de $2002^{9}$, se puede plantear que un niño con FQ está en riesgo nutricional si:

1. Aún estando eutrófico, presenta:

- Baja ponderal durante 1 mes o peso estacionario por 2 meses (en lactantes).

- Baja ponderal durante 2 meses o peso estacionario por 3 meses (en $>2$ años).

2. Tiene un crecimiento estatural sub-óptimo, considerando su potencial genético.

3. Presenta retraso puberal: Tanner I mamas en niñas de $\geq 13$ años, ausencia de menarquia con $\geq 16$ años o Tanner I en hombres $\geq 14$ años.

En estas situaciones debiera optimizarse su
Tabla 3. Otras causas de mal progreso ponderal (diferentes a la ingesta alimentaria insuficiente) en niños con $\mathbf{F Q}$

\begin{tabular}{|c|c|}
\hline Mecanismos & Causas \\
\hline Respiratorias & $\begin{array}{l}\text { Sobre infección pulmonar/sinusal (No } \\
\text { controlada o subclínica) } \\
\text { Hipoxia nocturna }\end{array}$ \\
\hline Gastrointestinales & $\begin{array}{l}\text { Esteatorrea persistente (Administración } \\
\text { incorrecta de enzimas, dosis insuficiente, } \\
\text { enzimas en mal estado...) } \\
\text { Reflujo gastroesofágico con control defi- } \\
\text { ciente } \\
\text { Sobrepoblación bacteriana intestinal } \\
\text { Intolerancia a la lactosa, enfermedad } \\
\text { celíaca, alergia a la proteína de leche de } \\
\text { vaca, enfermedad inflamatoria intestinal }\end{array}$ \\
\hline Otras & $\begin{array}{l}\text { Diabetes asociada a FQ } \\
\text { Pérdida exagerada de sodio en lactantes } \\
\text { Anemia ferropriva }\end{array}$ \\
\hline
\end{tabular}

Adaptada de: Guías clínicas Hospital Royal Brompton, en www.rbht.nhs.uk/childrencf

alimentación por vía oral ${ }^{8} \mathrm{y}$ descartarse otras posibles causas de mal progreso (tabla 3 ). Cuando existe desnutrición aguda leve $(\mathrm{P} / \mathrm{T} 90$ a $85 \%$ o IMC Pc 5-10) o retraso estatural (T/E $<-2 \mathrm{DS}$ o $<\mathrm{Pc5}$ ), debe realizarse apoyo más intensivo con uso de suplementos ${ }^{4,12}$ y con plazos acotados de espera, como los sugeridos en el flujograma (figura 4). La vía enteral es recomendable si no hay respuesta o si se produce desnutrición moderada o severa $(\mathrm{P} / \mathrm{T}<85 \%$ o $<$ $75 \%$ o IMC persiste $<$ pc 5$)^{8}$, para ello se requiere de una familia y paciente informados y motivados, del mejor control respiratorio posible y ojalá de apoyo psicológico.

\section{Implementación práctica}

La educación a la familia y los pacientes favorece una mejor adherencia al tratamiento ${ }^{11}$ y debe realizarse desde el momento del diagnóstico, destacando la importancia del estado nutricional en el pronóstico de la enfermedad. También debe existir anticipación a las posibles intervenciones a realizar en el curso de la enfermedad, en forma clara, cercana y positiva ${ }^{9}$.

Durante el primer año de vida la lactancia materna (LM) es óptima y puede ser beneficiosa para una mejor evolución de la $\mathrm{FQ}^{38}$. Es necesario el amamantamiento frecuente y ha- 


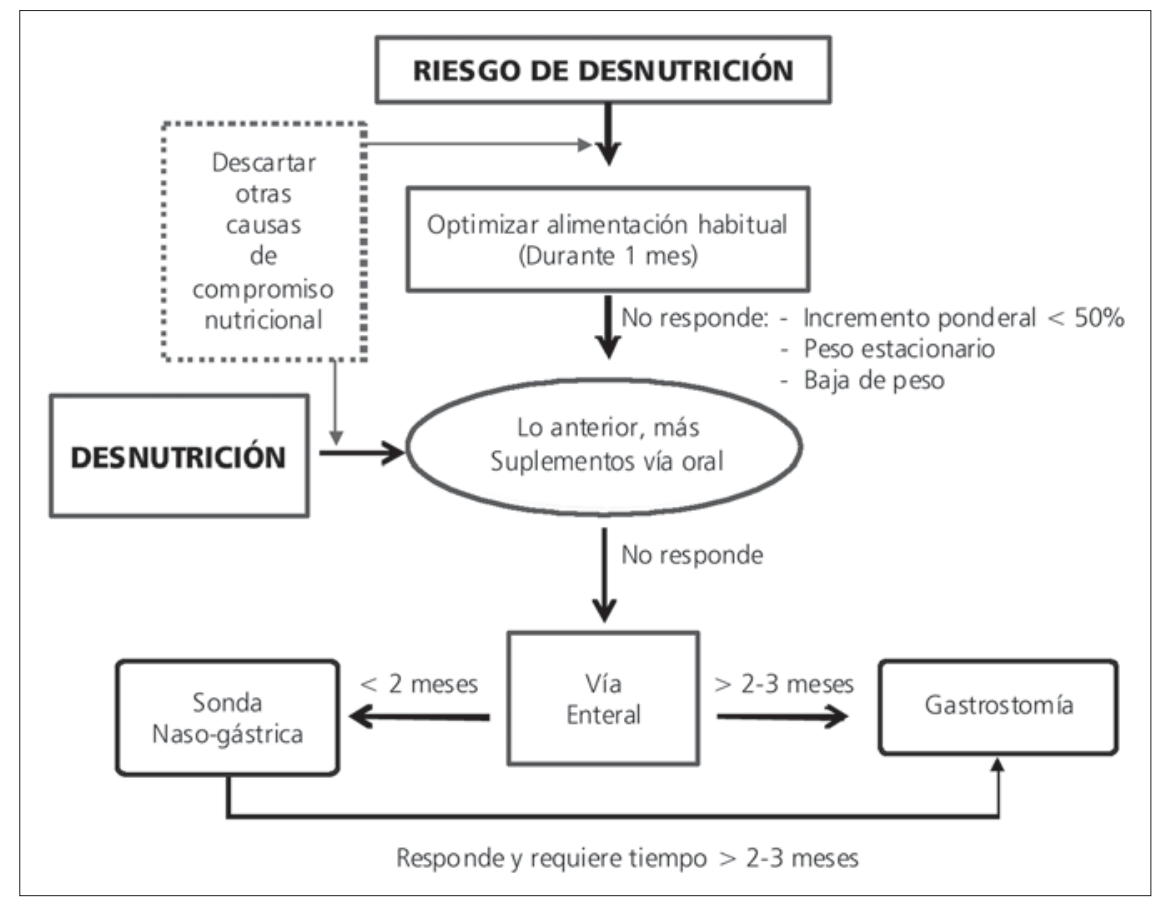

Figura 4. Flujograma sugerido para el manejo nutricional en niños con Fibrosis Quística. bitualmente fortificar la LM extraída. Si se requiere el uso de fórmulas lácteas de inicio, puede aumentarse aportes incrementando la concentración y adicionando malto-dextrina, aceite vegetal y/o módulo proteico, (aportar 1 $\mathrm{kcal} / \mathrm{ml}$, cuidando de mantener un porcentaje de calorías proteicas, o $\mathrm{P} \%$, adecuado). $\mathrm{La}$ alimentación complementaria se incorpora como es habitual, a los 6 meses de edad, considerando agregado controlado de sal y aumento del aporte calórico, si se requiere.

\section{Fortificación de la alimentación}

En general, la optimización de la alimentación habitual es el primer paso para incrementar su valor calórico y/o proteico ${ }^{7-9}$. En el niño mayor a dos años, es importante establecer cuatro comidas fijas, con adición de una o dos colaciones si es necesario, recordando que toda alimentación debe ser precedida por la dosis indicada de enzimas, salvo escasas excepciones. Se puede fortificar guisos y comidas con aceites vegetales (maíz, canola, maravilla, soya) o marinos (Ricos en AG Omega 3), con porciones controladas de alimentos ricos en lípidos, con fuentes proteicas naturales (cárneos, lác- teos) y carbohidratos de preferencia complejos (almidones, cereales).

\section{Suplementos nutricionales}

Para su indicación, es necesario considerar la edad, los requerimientos y la función gastrointestinal. El primer paso es su adición a la alimentación habitual. Su uso aumenta la ingesta, pero no siempre se asocia a mejoría nutricional en pacientes con menos compromiso nutricional, debido a que éstos reemplazan su alimentación habitual por los suplementos ${ }^{39}$.

1. Los suplementos calóricos habituales son malto-dextrinas (3-7\%) utilizadas con frecuencia para aumentar aportes en las comidas y los triglicéridos de cadena media (TCM, $1-2 \%$ ) que tienen absorción facilitada, pero son de alto costo económico y no aportan ácidos grasos de cadena larga (AGCL) ni esenciales (AGE), están indicados en presencia de compromiso hepático.

2. Los suplementos proteicos, como el Caseinato de Calcio (1-3\%), aumentan el aporte proteico, pero tienden a producir constipación y su aceptación puede ser limitada.

3. Las fórmulas poliméricas se pueden utilizar 
vía oral, inicialmente como complemento antes de acostarse, o como colación matinal, sin reemplazar la alimentación habitual. Son preparados líquidos o en polvo, de baja viscosidad, alta densidad calórica $(1 \mathrm{cal} / \mathrm{ml})$ e isotónicas, balanceadas en macro-nutrientes, con vitaminas y minerales. Se indican al $16-18 \%$ en el menor de un año de edad y al $20-22 \%$ en niños mayores, sobre los 10 años de edad es recomendable la formulación para adultos. Las Fórmulas Semi-Elementales y elementales son preparados líquidos de fácil absorción, de uso excepcional, en insuficiencia pancreática severa, mientras se ajusta el aporte enzimático, o en pacientes con Síndrome de Intestino Corto de difícil manejo. Tienen menor densidad calórica, mayor osmolalidad, macronutrientes más simples, minerales y vitaminas. En la mayoría de los pacientes, las fórmulas poliméricas, vía enteral, con infusión continua y adecuado aporte enzimático, pueden ser absorbidas de manera comparable a las elementales ${ }^{40}$.

4. Suplementos de vitaminas: Recomendaciones de aportes de vitaminas liposolubles y formulaciones disponibles, en tabla 4.
5. Suplementos de electrolitos: Los lactantes con FQ tienen riesgo de deshidratación hiponatrémica por sudoración excesiva y baja ingesta de agua, frente a climas calurosos, abrigo excesivo, fiebre o taquipnea ${ }^{7-9}$. Los requerimientos de Sodio son de 2-4 $\mathrm{mEq} / \mathrm{kg} /$ día para lactantes y se recomienda su aporte preventivo con 1/8 cucharadita de $\mathrm{NaCl}$ (Aprox. $11 \mathrm{mEq}$ de Sodio) o sales de rehidratación oral, para dar aportes más precisos.

6. Suplementos de minerales: Las recomendaciones de Calcio son las mismas que para la población general, se justifica suplementar si la dieta es insuficiente en aportar los requerimientos, dosis recomendada: 500$1300 \mathrm{mg} /$ día en escolares y adolescentes. Se suplementa Hierro en caso de déficit confirmado (administrar alejado de las enzimas pancreáticas y de las alimentaciones). El tratamiento con Zinc durante 6 meses es empírico, en falla de crecimiento, baja estatura o status sub óptimo de vitamina $\mathrm{A}^{8}$, con dosis de 5-10 mg de Zinc por día.

7. No se recomienda la suplementación rutinaria de AGE.

Tabla 4. Recomendaciones* y formulación de vitaminas para pacientes con Fibrosis Quística

\begin{tabular}{|c|c|c|c|c|}
\hline Edad & Vitamina A (UI) & Vitamina E (UI) & Vitamina D (UI) & Vitamina K (mg) \\
\hline $0-12$ meses & 1500 & $40-50$ & 400 & $0,3-0,5$ \\
\hline $1-3$ años & 5000 & $80-150$ & $400-800$ & $0,3-0,5$ \\
\hline $4-8$ años & $5000-10000$ & $100-200$ & $400-800$ & $0,3-0,5$ \\
\hline \multirow[t]{2}{*}{$>8$ años } & 10000 & $200-400$ & $400-800$ & $0,3-0,5$ \\
\hline & $\begin{array}{c}\text { ADEKs }{ }^{\circledR} \\
\text { Gotas }(1 \mathrm{ml})\end{array}$ & $\begin{array}{l}\text { ADEKs }{ }^{\circledR} \\
\text { Tabletas }\end{array}$ & $\begin{array}{l}\text { AquADEKs }{ }^{\circledR} \\
\text { Gotas }(1 \mathrm{ml})\end{array}$ & $\begin{array}{l}\text { AquADEKs }{ }^{\circledR} \\
\text { Caps. soft-gel }\end{array}$ \\
\hline Vit. A (UI) & 1500 & 4000 & 5751 & 18167 \\
\hline Vit. D (UI) & 400 & 400 & 400 & 800 \\
\hline Vit. E (UI) & 40 & 150 & 50 & 150 \\
\hline Vit. K (mg) & 0,1 & 0,15 & 0,4 & 0,7 \\
\hline Vit C (mg) & 45 & 60 & 45 & 75 \\
\hline$\beta$ Caroteno (mg) & 1 & 3 & 3 & 10 \\
\hline Zinc (mg) & 5 (Sulfato) & 7,5 (Oxido) & 5 (Gluconato) & 10 (Sulfato) \\
\hline Otros & $\begin{array}{c}\mathrm{B} 1, \mathrm{~B} 2, \mathrm{~B} 3, \mathrm{~B} 5 \\
\mathrm{~B} 6, \mathrm{~B} 8, \mathrm{~B} 12\end{array}$ & $\begin{array}{l}\text { B1, B2, B3, B5, B6, B8, } \\
\text { B12, Ácido Fólico, Q10 }\end{array}$ & $\begin{array}{c}\text { B1, B2, B3, B5, B6, } \\
\text { B8, B12 Se, Q10 }\end{array}$ & $\begin{array}{l}\text { B1, B2, B3, B5, B6, B8, } \\
\text { B12, Acido Fólico, Q10 }\end{array}$ \\
\hline Prescripción & $\begin{array}{l}\text { 0-12 m: } 1 \mathrm{ml} / \mathrm{día} \\
1-3 \text { a: } 2 \mathrm{ml} / \mathrm{día}\end{array}$ & $\begin{array}{l}\text { 4-10 a: } 1 / \text { día } \\
>10 \text { a: } 2 / \text { día }\end{array}$ & $\begin{array}{c}\text { 0-12 m: } 1 \mathrm{ml} / \mathrm{día} \\
\text { 1-3 a: } 2 \mathrm{ml} / \mathrm{d} i ́ a\end{array}$ & $\begin{array}{l}\text { 4-10 a: 1/día } \\
>10 \text { a: 2/día }\end{array}$ \\
\hline
\end{tabular}

*Adaptado de Borowitz D y cols, $2002^{9}$ 


\section{Apoyo nutricional por vía enteral}

Existe bastante evidencia del beneficio nutricional del aporte vía enteral en niños con $\mathrm{FQ}^{4,41}$, sin embargo, debido a la indicación tardía, no se ha demostrado suficiente efecto en la evolución de la enfermedad, sujeta además a múltiples otras variables ${ }^{42}$.

\section{Vía de aporte}

La sonda naso-gástrica es apropiada para períodos menores a dos meses, como en hospitalizaciones, compromiso nutricional secundario a exacerbación u otra causa reversible en su efecto sobre la ingesta. Es de fácil instalación, pero baja tolerancia, dificultad de mantener en posición, riesgo de aspiración y complicaciones locales en uso prolongado. Las sondas nasoyeyunales son mejor toleradas, pero se obstruyen fácilmente, son difíciles de mantener en posición y requieren uso de bomba enteral. El método más apropiado para mantener la vía enteral por un período mayor a 3 meses es la gastrostomía y en forma muy infrecuente, la yeyunostomía.

\section{Condiciones para inicio de alimentación enteral}

Es recomendable realizar previamente estudio de reflujo gastroesofágico y test de tolerancia oral a la glucosa. La instalación de la sonda requiere idealmente de hospitalización, para monitorización y educación familiar. El inicio de la alimentación y su progresión deben ser graduales, habitualmente utilizando fórmulas poliméricas. De preferencia, aportar el 30-50\% de los requerimientos diarios a través de alimentación enteral continua durante la noche y mantener pauta habitual durante el día ${ }^{8,9}$. Se recomienda fraccionar las enzimas pancreáticas en $50 \%$ al inicio y $50 \%$ a la mitad de la alimentación.

\section{Gastrostomía en Fibrosis Quística}

En un $3-10 \%$ de los pacientes con FQ se requiere realizar gastrostomía ${ }^{17}$; se ha demostrado que ésta permite mejorar el estado nutricional y al menos disminuir el deterioro de la función pulmonar ${ }^{41,43}$. Por ello, la tendencia actual es a utilizarla en forma más precoz, para lo cual existe evidencia parcial en cuanto a disminución de la morbimortalidad, de manera que se requieren estudios controlados y de mayor tiempo de seguimiento, en especial cuando el procedimiento se indique en pacientes con compromiso nutricional menos avanzado ${ }^{42}$.

\section{Nutrición parenteral}

La nutrición parenteral constituye un método eficaz para mejorar el estado nutricional en pacientes desnutridos con $\mathrm{FQ}$, pero por ser un método invasivo no exento de complicaciones, su uso se limita a situaciones específicas, en las cuales no pueda usarse la vía enteral o ser ésta insuficiente, por períodos cortos, por ejemplo, en cirugías, trasplante de pulmón o hígado o en Síndrome de Intestino Corto ${ }^{7,8}$.

\section{Referencias}

1.- Bell SC, Shepherd RW: Optimizing nutrition in cystic fibrosis. J Cyst Fibros 2002; 1: 47-50.

2.- Cystic Fibrosis Foundation: Patient Registry 2006 Annual Report, Bethesda, Maryland.

3.- Sánchez I, Pérez MA H, Boza L, et al: Consenso nacional de Fibrosis Quística. Rev Chil Pediatr 2001; 72: 356-80.

4.- Stallings, VA, Stark LJ, Robinson KA, Feranchack A, Quinton H: Evidence-Based Practice. Recommendations for Nutrition-Related Management of Children and Adults with Cystic Fibrosis and Pancreatic Insufficiency: Results of a Systematic Review: clinical practice guidelines on growth and nutrition subcommittee; ad hoc working group. J Am Diet Assoc 2008; 108: 832-9

5.- Corey M, McLaughlin FJ, Williams M, Levison H: A comparison of survival, growth, and pulmonary function in patients with cystic fibrosis in Boston and Toronto. J Clin Epidemiol 1988; 41 (6): 583-91.

6.- Sharma R, Florea VG, Bolger AP, et al: Wasting as an independent predictor of mortality in patients with cystic fibrosis. Thorax 2001; 56: 746-50.

7.- Ramsey BW, Farrell PM, Pencharz P, et al: Nutritional assessment and management in cystic fibrosis: a consensus report. The Consensus Committee. Am J Clin Nutr 1992; 55: 108-16.

8.- Sinaasappel M, Stern M, Littlewood J, et al: Nutrition in patients with cystic fibrosis: a European Consensus. J Cyst Fibros 2002; 1: 51-75.

9.- Borowitz D, Baker RD, Stallings $V$, et al: Consensus report on nutrition for pediatric patients with cystic fibrosis. J Pediatr Gastroenterol Nutr 2002; 35: 24659.

10.- Dodge JA, Turk D: Cystic fibrosis: nutritional consequences and management. Best Pract Res Clin Gastroenterol 2006; 20: 531-46. 
11.- Adde F, Rodrigues J, Cardosos A: Nutritional followup of CF patients: The role of nutrition education. $J$ Pediatr (Rio) 2004; 80: 475-82.

12.- Barja S, Rebollo MJ, Reyes $M$, González $M$, Chávez E, Jofré P: Comisión Técnica, Programa Nacional de Fibrosis Quística: Manual técnico de diagnóstico y tratamiento de la Fibrosis Quística: Aspectos nutricionales y gastroenterológicos. En: www.fibrosisquisticachile.cl

13.- Reis F, Oliveira M, Penna F, et al: Quadro clínico e nutricional de pacientes com Fibrose Cística: 20 anos de seguimento no HC-UFMG. Rev Med Brasil 2000; 46: 325-30.

14.- Wiedemann B, Paul KD, Stern M, Wagner TO, Hirshe TO: Evaluation of body mass index percentiles for assessment of malnutrition in children with CF. Eur J of Clin Nutr 2007; 61: 759-68.

15.- Moudiou T, Galli-Tsinopoulou A, Vamvakoudis E, Nousia-Arvanitakis S: Resting energy expenditure in cystic fibrosis as an indicator of disease severity. $\mathrm{J}$ Cyst Fibros 2006; doi:10.1016/j.jcf.2006.06.001.

16.- Marín V, Velandia S, Hunter B, et al: Energy Expenditure, Nutrition Status, and Body Composition in Children With Cystic Fibrosis. Nutrition 2004; 20: 181-6.

17.- Pencharz PB, Durie PR: Pathogenesis of malnutrition in Cystic Fibrosis and its treatment. Clinical Nutrition 2000; 19: 387-94.

18.- Trabulsi I, Ihenbach R F, Shall Jl, et al: Evaluation of formulas for calculating total energy requirements of preadolescent and children with Cystic fibrosis. Am J Clin Nutr 2007; 85: 144-51.

19.- White H, Wolfe S, Foy J, Morton A, Conway S, Brownlee $K$ : Nutritional Intake and Status in Children With Cystic Fibrosis: Does Age Matter? JPGN 2007; 44: 116-23.

20.- Anderson C, Al-Turkmani MR, Savaille JE, et al: Cell culture models demonstrate that CFTR dysfunction leads to defective fat composition and metabolism. J Lipid Res 2008; 49: 1692-700.

21.- Colombo C, Bennato B, Costantini D, et al: Dietary and circulating polyunsaturated fatty acids in Cystic fibrosis: are they related to clinical outcomes? J Pediatr Gastroenterol Nutr 2006; 43: 660-5.

22.- Maqbool A, Schall JI, García-Espana JF, Zemel BS, Strandvik, Stallings VA: Serum linoleic acid status as a clinical indicator of essential fatty acid status in children with cystic fibrosis. J Pediatr Gastroenterol Nutr 2008; 47: 635-44.

23.- Panchand A, Sauly A, Kernen Y, et al: Biological effects of a dietary omega-3 polyunsaturated fatty acids supplementation in cystic fibrosis patients: a randomized, crossover placebo-controlled trial. Clin Nutr 2006; 25: 418-27.

24.- De Vizia B, Raia V, Spano C, Pavlidis C, Corruzo A, Alessio M: Effect of an 8 month treatment with omega-3 fatty acids EPA and DHA in patients with Cystic Fibrosis. JPEN 2003; 27: 52-7.

25.- Van Biervliet $S$, Devos M, Delhaye T, Van Biervliet $J P$, Robberecht E, Christophe A: Oral DHA supple- mentation in delta F508 homozygous Cystic fibrosis patients. Prostaglandins leukot Essent Fatty Acids 2008; 78: 109-15.

26.- Rovner AJ, Stallings VA, Schall JL, Leonard MB, Zemel BS: Vitamin D insufficency in children, adolescents and young adults with cystic fibrosis despite rutine oral supplementation. Am J Clin Nutr 2007; 86: 1694-9.

27.- Aris R, Lester G, Ontjes D: Treatment of bone disease in cystic fibrosis. Curr Opin Pulm Med 2004; 10: 52430.

28.- Aris R, Merkel P, Bachrach L, et al: Consensus Statement: Guide to Bone Health and Disease in Cystic Fibrosis. J Clin Endocrinol Metab 2005; 90: 1888-96.

29.- Ujhelyi R, Tresz A, Vásárhelyi B, et al: Bone Mineral Density and Bone Acquisition in Children and Young Adults with Cystic Fibrosis: A Follow-up Study. JPGN 2004; 38: 401-6.

30.- Conway SP, Wolfe SP, Brownlee KG, et al: Vitamin K status among children with cystic fibrosis and its relationship to bone mineral density and bone turnover. Pediatrics 2005; 115: 1325-31.

31.- Haskim F, Kerem E, Rivlin J, et al: Vitamins A, E and pulmonary exacerbations in patients with cystic fibrosis. J Pediatr Gastroenterol Nutr 2007; 45: 34753.

32.- Schulze K, O'Brien K, Germain-Lee E, Baer D, Leonard A, Rosenstein B: Efficiency of calcium absorption is not compromised in clinically stable prepubertal and pubertal girls with cystic fibrosis. Am J Clin Nutr 2003; 78: 110-6.

33.- Reid DW, Withers NJ, Francis LRN, Wilson JW, Kotsimbos TC: Iron Deficiency in Cystic Fibrosis: Relationship to Lung Disease Severity and Chronic Pseudomonas Aeruginosa Infection. CHEST 2002; 121: 48-54.

34.- Akanli L, Lowenthal D, Gjonaj S, Dozor AJ: Plasma and Red Blood Cell Zinc in Cystic Fibrosis. Pediatr Pulmonol 2003; 35: 2-7.

35.- Abdulhamid I, Beck FW, Millard S, Chen X, Prasad A: Effect of Zinc Supplementation on Respiratory Tract Infections in Children With Cystic Fibrosis. Pediatr Pulmonol 2008; 43: 281-7.

36.- Akbar A, Rees JHM, Nyamugunduru G, English MW, Spencer DA, Weller PH: Aminoglycoside-associated hypomagnesaemia in children with cystic fibrosis. Acta Paediatr 1999; 88: 783-5.

37.- Sanders N, Franckx H, De Boeck K, Haustraete J, De Smedt SC, Demeester J: Role of magnesium in the failure of rhDNase therapy in patients with cystic fibrosis. Thorax 2006; 61: 962-8. doi: 10.1136/ thx.2006.060814.

38.- Colombo C, Costantini D, Zazzeron L, et al: Benefits of breastfeeding in cystic fibrosis: A single-centre follow-up survey. Acta Pædiatrica 2007; 96: 122832.

39.- Smyth R, Walters $S$ : Oral calorie supplements for cystic fibrosis. Cochrane Database of Systematic Reviews 2007, Issue1.Art. No: CD000406. DOI: 10.1002/ 14651858. CD000406.pub2. 
BARJA S. y col.

40.- Erskine JM, Lingard CD, Sontag MK, Accurso FJ: Enteral nutrition for patients with cystic fibrosis: comparison of a semi-elemental and no elemental formula. J Pediatr 1998; 132: 265-9.

41.- Kalnins D, Corey M, Ellis L, Pencharz PB, Tullis E, Durie PR: Failure of conventional strategies to improve nutritional status in malnourished adolescents and adults with cystic fibrosis. J Pediatr 2005; 147 : 399-401.
42.- Conway SP, Morton A, Wolfe S: Enteral tube feeding for cystic fibrosis. Cochrane Database of Systematic Reviews 2008, Issue2. Art.No. CD001198.DOI: 10.1002/14651858.CD001198.pub2.

43.- Efrati O, Mei-Zahav M, Rivlin J, et al: Long Term Nutritional Rehabilitation by Gastrostomy in Israeli Patients with Cystic Fibrosis: Clinical Outcome in Advanced Pulmonary Disease. JPGN 2006; 42: 2228 